TAIWANESE JOURNAL OF MATHEMATICS

Vol. 18, No. 4, pp. 1003-1019, August 2014

DOI: $10.11650 /$ tjm.18.2014.2678

This paper is available online at http://journal.taiwanmathsoc.org.tw

\title{
ASYMPTOTIC BEHAVIOR OF FOURTH-ORDER NEUTRAL DYNAMIC EQUATIONS WITH NONCANONICAL OPERATORS
}

\author{
Tongxing Li*, Chenghui Zhang and Ethiraju Thandapani
}

Abstract. This paper is concerned with asymptotic behavior of a class of fourthorder neutral delay dynamic equations with a noncanonical operator on an arbitrary time scale. A new asymptotic criterion and an illustrative example are included.

\section{INTRODUCTION}

In this paper, we study asymptotic properties of a fourth-order neutral delay dynamic equation

$$
L z+q(t) x(\delta(t))=0, \quad L z:=\left(r z^{\Delta^{3}}\right)^{\Delta}(t)
$$

on an arbitrary time scale $\mathbb{T}$ with $\sup \mathbb{T}=\infty$, where

$$
z(t):=x(t)+p(t) x(\tau(t)) .
$$

Since our concern is asymptotic behavior of solutions, we assume the time scale interval is the form $\left[t_{0}, \infty\right)_{\mathbb{T}}:=\left[t_{0}, \infty\right) \cap \mathbb{T}$. Throughout, we assume $r, q \in \mathrm{C}_{\mathrm{rd}}\left(\left[t_{0}, \infty\right)_{\mathbb{T}},(0, \infty)\right)$, $p \in \mathrm{C}_{\mathrm{rd}}\left(\left[t_{0}, \infty\right)_{\mathbb{T}}, \mathbb{R}\right), 0 \leq p(t) \leq p_{1}<1, \tau, \delta \in \mathrm{C}_{\mathrm{rd}}\left(\left[t_{0}, \infty\right)_{\mathbb{T}}, \mathbb{T}\right), \tau(t) \leq t, \delta(t) \leq t$, and $\lim _{t \rightarrow \infty} \tau(t)=\lim _{t \rightarrow \infty} \delta(t)=\infty$. The operator $L z$ (given in (1.1)) is said to be in noncanonical form if

$$
\int_{t_{0}}^{\infty} \frac{\Delta t}{r(t)}<\infty
$$

By a solution of (1.1) we mean a function $x \in \mathrm{C}_{\mathrm{rd}}\left[T_{x}, \infty\right)_{\mathbb{T}}, T_{x} \in\left[t_{0}, \infty\right)_{\mathbb{T}}$, which has the property $r z^{\Delta^{3}} \in \mathrm{C}_{\mathrm{rd}}^{1}\left[T_{x}, \infty\right)_{\mathbb{T}}$ and satisfies $(1.1)$ on $\left[T_{x}, \infty\right)_{\mathbb{T}}$. We consider only those solutions $x$ of (1.1) which satisfy $\sup \{|x(t)|: t \in[T, \infty) \mathbb{T}\}>0$ for all

Received November 19, 2012, accepted December 5, 2013.

Communicated by Eiji Yanagida.

2010 Mathematics Subject Classification: 34K11, 34N05, 39A10.

Key words and phrases: Asymptotic behavior, Fourth-order delay equation, Neutral dynamic equation, Time scale.

*Corresponding author. 
$T \in\left[T_{x}, \infty\right)_{\mathbb{T}}$ and assume that (1.1) possesses such solutions. A solution of (1.1) is called oscillatory if it is neither eventually positive nor eventually negative; otherwise, it is called nonoscillatory.

Fourth-order differential equations are quite often encountered in mathematical models of various physical, biological, and chemical phenomena. Applications include, for instance, problems of elasticity, deformation of structures, or soil settlement; see [8].

In mechanical and engineering problems, questions related to the existence of oscillatory and nonoscillatory solutions play an important role. As a result, there has been much activity concerning oscillatory and asymptotic behavior of various classes of differential and difference equations; see, e.g., [7, 8, 39, 41] and [6, 12, 34-36], and the references cited therein. Following the development of the theory of dynamic equations on time scales, oscillatory properties of various classes of equations on time scales has become an important area of research due to the fact that such equations arise in many real life problems $[9,10,19]$. We refer the reader to $[1-5,11,13-18$, $20-33,37,38,40]$ and the references cited therein. Thereinto, monotone and oscillatory behavior of solutions to a fourth-order dynamic equation

$$
\left(a\left(x^{\Delta^{2}}\right)^{\alpha}\right)^{\Delta^{2}}(t)+q(t) x^{\beta}(\sigma(t))=0
$$

with the property that

$$
\frac{x(t)}{\int_{t_{0}}^{t} \int_{t_{0}}^{s} a^{-1 / \alpha}(\tau) \Delta \tau \Delta s} \rightarrow 0 \quad \text { as } \quad t \rightarrow \infty
$$

were established by Grace et al. [16]. Grace et al. [15] studied a fourth-order dynamic equation

$$
x^{\Delta^{4}}(t)+q(t) x^{\gamma}(\sigma(t))=0 .
$$

Grace et al. [17] considered a fourth-order dynamic equation

$$
x^{\Delta^{4}}(t)+q(t) x^{\gamma}(t)=0 .
$$

$\mathrm{Li}$ et al. [25] investigated oscillation of unbounded solutions to a fourth-order delay dynamic equation

$$
\left(r x^{\Delta^{3}}\right)^{\Delta}(t)+q(t) x(\tau(t))=0
$$

under the assumption that (1.2) holds, and obtained some comparison theorems. Zhang et al. [40] studied a fourth-order dynamic equation

$$
\left(r x^{\Delta^{3}}\right)^{\Delta}(t)+q(t) f(x(\sigma(t)))=0
$$

in the case where (1.2) holds. Later, Wu et al. [38] extended results of [40] to a generalized fourth-order dynamic equation

$$
\left(r\left(a\left(b x^{\Delta}\right)^{\Delta}\right)^{\Delta}\right)^{\Delta}(t)+q(t) f(x(\sigma(t)))=0
$$


under the assumptions that (1.2) holds and $\int_{t_{0}}^{\infty} \frac{\Delta t}{a(t)}=\int_{t_{0}}^{\infty} \frac{\Delta t}{b(t)}=\infty$. However, there are few results dealing with asymptotic behavior of solutions to higher-order neutral dynamic equations [13, 18, 20-22, 26, 37]. Graef et al. [18], Panigrahi and Rami Reddy [26], and Thandapani et al. [37] studied a fourth-order neutral delay dynamic equation

$$
\left[r(t)(x(t)+p(t) x(\tau(t)))^{\Delta^{2}}\right]^{\Delta^{2}}+q(t) f(x(\delta(t)))=0
$$

in the cases $\int_{t_{0}}^{\infty} \frac{\sigma(t)}{r(t)} \Delta t<\infty$ or $\int_{t_{0}}^{\infty} \frac{t}{r(t)} \Delta t=\infty$. In particular, the authors in [13, 20-22] investigated a higher-order neutral dynamic equation

$$
[x(t)+p(t) x(\tau(t))]^{\Delta^{n}}+q(t) x(\delta(t))=0 .
$$

Note that the results given in [13, 20-22] can be applied to equation (1.1) in the case where $r(t)=1$. To the best of our knowledge, there are no known asymptotic criteria to cover equation (1.1) in the case where (1.2) holds. Therefore, the objective of this paper is to derive an asymptotic criterion for this equation assuming that (1.2) holds.

\section{MAIN Results}

In what follows, all functional inequalities are assumed to hold eventually, that is, they are satisfied for all $t$ large enough. We begin with the following lemma.

Lemma 2.1. Assume that (1.2) holds and $x$ is an eventually positive solution of (1.1). Then there are the following four possible cases eventually:

(1) $z>0, \quad z^{\Delta}<0, \quad z^{\Delta^{2}}>0, \quad z^{\Delta^{3}}<0, \quad\left(r z^{\Delta^{3}}\right)^{\Delta}<0 ;$

(2) $z>0, \quad z^{\Delta}>0, \quad z^{\Delta^{2}}>0, \quad z^{\Delta^{3}}<0, \quad\left(r z^{\Delta^{3}}\right)^{\Delta}<0$;

(3) $z>0, \quad z^{\Delta}>0, \quad z^{\Delta^{2}}>0, \quad z^{\Delta^{3}}>0, \quad\left(r z^{\Delta^{3}}\right)^{\Delta}<0$;

(4) $z>0, \quad z^{\Delta}>0, \quad z^{\Delta^{2}}<0, \quad z^{\Delta^{3}}>0, \quad\left(r z^{\Delta^{3}}\right)^{\Delta}<0$.

Proof. Let $x$ be an eventually positive solution of (1.1) on $\left[t_{0}, \infty\right)_{\mathbb{T}}$. Then there exists a $t_{1} \in\left[t_{0}, \infty\right)_{\mathbb{T}}$ such that $x(t)>0, x(\tau(t))>0$, and $x(\delta(t))>0$ for $t \in\left[t_{1}, \infty\right)_{\mathbb{T}}$. Hence, $z>0$ eventually. By virtue of (1.1), we have

$$
\left(r z^{\Delta^{3}}\right)^{\Delta}(t)=-q(t) x(\delta(t))<0
$$

for $t \in\left[t_{1}, \infty\right)_{\mathbb{T}}$. Thus, $r z^{\Delta^{3}}$ is decreasing. Then $z^{\Delta}, z^{\Delta^{2}}$, and $z^{\Delta^{3}}$ are of constant sign eventually. Assume first that $z^{\Delta^{3}}<0$. Then $z^{\Delta^{2}}>0$. If not, then $z<0$ eventually when using $z^{\Delta}<0$ and $z^{\Delta^{2}}<0$, which is a contradiction. Hence, there are possible cases (1) and (2). Assume now that $z^{\Delta^{3}}>0$ and $z^{\Delta^{2}}>0$. Then $z^{\Delta}>0$. 
If $z^{\Delta^{3}}>0$ and $z^{\Delta^{2}}<0$, then $z^{\Delta}>0$ (since $z^{\Delta}<0$ and $z^{\Delta^{2}}<0$ imply that $z<0$ ). Hence, there are possible cases (3) and (4). The proof is complete.

In [9, Section 1.6], the Taylor monomials $\left\{h_{n}(t, s)\right\}_{n=0}^{\infty}$ are defined recursively by

$$
h_{0}(t, s)=1, \quad h_{n+1}(t, s)=\int_{s}^{t} h_{n}(\tau, s) \Delta \tau, \quad t, s \in \mathbb{T}, \quad n \geq 0 .
$$

One has $h_{1}(t, s)=t-s$ for any time scale, but simple formulas in general do not hold for $n \geq 2$.

Now we present the main results. We use the notation

$$
R(t):=\int_{t}^{\infty} \frac{\Delta s}{r(s)} \quad \text { and } \quad \pi_{+}(t):=\max \{0, \pi(t)\} .
$$

Theorem 2.2. Assume (1.2) and let

$$
\begin{aligned}
\limsup _{t \rightarrow \infty} \int_{t_{0}}^{t} & {\left[b_{0} q(v) \int_{\sigma(v)}^{\infty} \int_{u}^{\infty} R(s) \Delta s \Delta u\right.} \\
& \left.-\frac{\int_{v}^{\infty} R(s) \Delta s}{4 \int_{\sigma(v)}^{\infty} \int_{u}^{\infty} R(s) \Delta s \Delta u}\right] \Delta v=\infty
\end{aligned}
$$

hold for all constants $b_{0}>0$. Suppose further that

$$
\begin{aligned}
\limsup _{t \rightarrow \infty} \int_{t_{0}}^{t} & {\left[q(s) R(\sigma(s))(1-p(\delta(s))) \frac{h_{2}\left(\delta(s), t_{0}\right)}{2}\right.} \\
& \left.-\frac{1}{4 r(s) R(\sigma(s))}\right] \Delta s=\infty .
\end{aligned}
$$

If there exist two positive functions $\alpha, \beta \in \mathrm{C}_{\mathrm{rd}}^{1}\left(\left[t_{0}, \infty\right)_{\mathbb{T}}, \mathbb{R}\right)$ such that

$$
\begin{aligned}
\limsup _{t \rightarrow \infty} \int_{t_{4}}^{t} & {\left[\alpha(\sigma(s)) q(s)(1-p(\delta(s))) \frac{\int_{t_{3}}^{\delta(s)} \int_{t_{2}}^{\eta} \int_{t_{1}}^{v} \frac{1}{r(u)} \Delta u \Delta v \Delta \eta}{\int_{t_{1}}^{\sigma(s)} \frac{1}{r(u)} \Delta u}\right.} \\
& \left.-\frac{r(s)\left(\alpha_{+}^{\Delta}(s)\right)^{2}}{4 \alpha(\sigma(s))} \frac{\int_{t_{1}}^{\sigma(s)} \frac{1}{r(u)} \Delta u}{\int_{t_{1}}^{s} \frac{1}{r(u)} \Delta u}\right] \Delta s=\infty
\end{aligned}
$$


holds for all sufficiently large $t_{1} \in\left[t_{0}, \infty\right)_{\mathbb{T}}$ and for $t_{4}>t_{3}>t_{2}>t_{1}$, and

$$
\begin{aligned}
\limsup _{t \rightarrow \infty} \int_{t_{0}}^{t} & {\left[k^{2} \beta(\sigma(s)) \frac{s}{\sigma(s)} \int_{s}^{\infty} \frac{1}{r(u)} \int_{u}^{\infty} q(v)(1-p(\delta(v))) \frac{\delta(v)}{v} \Delta v \Delta u\right.} \\
& \left.-\frac{\sigma(s)\left(\beta_{+}^{\Delta}(s)\right)^{2}}{4 k s \beta(\sigma(s))}\right] \Delta s=\infty
\end{aligned}
$$

holds for some $k \in(0,1)$, then every solution $x$ of (1.1) is either oscillatory or satisfies $\lim _{t \rightarrow \infty} x(t)=0$.

Proof. Suppose that (1.1) has a nonoscillatory solution $x$. We may assume without loss of generality that there exists a $t_{1} \in\left[t_{0}, \infty\right)_{\mathbb{T}}$ such that $x(t)>0, x(\tau(t))>0$, and $x(\delta(t))>0$ for all $t \in\left[t_{1}, \infty\right)_{\mathbb{T}}$. From Lemma 2.1, we get that $z$ satisfies four possible cases. In the following, we consider each of four cases separately.

Assume (1). By virtue of $z^{\Delta^{2}}>0$ and $z^{\Delta^{3}}<0$, there exists a constant $c_{0} \geq 0$ such that

$$
\lim _{t \rightarrow \infty} z^{\Delta^{2}}(t)=c_{0} \geq 0
$$

Using the fact that $r z^{\Delta^{3}}$ is decreasing, we have

$$
r(s) z^{\Delta^{3}}(s) \leq r(t) z^{\Delta^{3}}(t), \quad s \in[t, \infty)_{\mathbb{T}} .
$$

Dividing the latter inequality by $r(s)$ and integrating the resulting inequality from $t$ to $l$, we obtain

$$
z^{\Delta^{2}}(l) \leq z^{\Delta^{2}}(t)+r(t) z^{\Delta^{3}}(t) \int_{t}^{l} \frac{\Delta s}{r(s)} .
$$

Passing to the limit as $l \rightarrow \infty$ and using (2.5), we deduce that

$$
z^{\Delta^{2}}(t) \geq-r(t) z^{\Delta^{3}}(t) R(t) .
$$

From $z^{\Delta}<0$ and $z^{\Delta^{2}}>0$, there exists a constant $c_{1} \leq 0$ such that

$$
\lim _{t \rightarrow \infty} z^{\Delta}(t)=c_{1} \leq 0
$$

Integrating (2.6) from $t$ to $\infty$ and using (2.7), we have

$$
-z^{\Delta}(t) \geq \int_{t}^{\infty}-r(s) z^{\Delta^{3}}(s) R(s) \Delta s \geq-r(t) z^{\Delta^{3}}(t) \int_{t}^{\infty} R(s) \Delta s .
$$

Integrating (2.8) from $t$ to $\infty$, we get

$$
\begin{aligned}
z(t) & \geq \int_{t}^{\infty}-r(u) z^{\Delta^{3}}(u) \int_{u}^{\infty} R(s) \Delta s \Delta u \\
& \geq-r(t) z^{\Delta^{3}}(t) \int_{t}^{\infty} \int_{u}^{\infty} R(s) \Delta s \Delta u .
\end{aligned}
$$


It follows from $z>0$ and $z^{\Delta}<0$ that there exists a constant $a_{0} \geq 0$ such that $\lim _{t \rightarrow \infty} z(t)=a_{0}$. Next, we consider two cases. If $a_{0}>0$, then for any $\varepsilon>0$, we have $a_{0}+\varepsilon>z(t)>a_{0}-\varepsilon$ eventually. Choose $0<\varepsilon<a_{0}\left(1-p_{1}\right) /\left(1+p_{1}\right)$. It is not difficult to verify that

$$
x(t)=z(t)-p(t) x(\tau(t))>\left(a_{0}-\varepsilon\right)-p_{1}\left(a_{0}+\varepsilon\right)=b_{0}\left(a_{0}+\varepsilon\right)>b_{0} z(t),
$$

where $b_{0}:=\left(a_{0}-\varepsilon-p_{1}\left(a_{0}+\varepsilon\right)\right) /\left(a_{0}+\varepsilon\right)>0$. Hence, (1.1) implies that

$$
\left(r z^{\Delta^{3}}\right)^{\Delta}(t)+b_{0} q(t) z(\delta(t)) \leq 0 .
$$

Now set

$$
\omega(t):=\frac{r(t) z^{\Delta^{3}}(t)}{z(t)}, \quad t \in\left[t_{1}, \infty\right)_{\mathbb{T}}
$$

Then $\omega(t)<0$ for $t \in\left[t_{1}, \infty\right)_{\mathbb{T}}$ and we have

$$
\begin{aligned}
\omega^{\Delta}(t) & =\frac{\left(r z^{\Delta^{3}}\right)^{\Delta}(t)}{z(\sigma(t))}-\frac{r(t) z^{\Delta^{3}}(t) z^{\Delta}(t)}{z(t) z(\sigma(t))} \\
& \leq-b_{0} q(t) \frac{z(\delta(t))}{z(\sigma(t))}-\frac{r(t) z^{\Delta^{3}}(t) z^{\Delta}(t)}{z(t) z(\sigma(t))} \\
& \leq-b_{0} q(t)-\frac{r(t) z^{\Delta^{3}}(t) z^{\Delta}(t)}{z(t) z(\sigma(t))}
\end{aligned}
$$

due to (2.10), $z^{\Delta}<0$, and $\delta(t) \leq t$. Using (2.8), (2.11), (2.12), and $z^{\Delta}<0$, we have

$$
\begin{aligned}
\omega^{\Delta}(t) & \leq-b_{0} q(t)-\frac{\left(r z^{\Delta^{3}}\right)^{2}(t)}{z^{2}(t)} \int_{t}^{\infty} R(s) \Delta s \\
& =-b_{0} q(t)-\omega^{2}(t) \int_{t}^{\infty} R(s) \Delta s .
\end{aligned}
$$

In view of (2.9), we conclude that

$$
\omega(t) \int_{t}^{\infty} \int_{u}^{\infty} R(s) \Delta s \Delta u \geq-1
$$

From (2.13), we obtain

$$
\begin{aligned}
\omega^{\Delta}(t) \int_{\sigma(t)}^{\infty} \int_{u}^{\infty} R(s) \Delta s \Delta u \leq & -b_{0} q(t) \int_{\sigma(t)}^{\infty} \int_{u}^{\infty} R(s) \Delta s \Delta u \\
& -\omega^{2}(t) \int_{\sigma(t)}^{\infty} \int_{u}^{\infty} R(s) \Delta s \Delta u \int_{t}^{\infty} R(s) \Delta s .
\end{aligned}
$$


Integrating (2.15) from $t_{1}$ to $t$, one arrives at

$$
\begin{aligned}
& \omega(t) \int_{t}^{\infty} \int_{u}^{\infty} R(s) \Delta s \Delta u-\omega\left(t_{1}\right) \int_{t_{1}}^{\infty} \int_{u}^{\infty} R(s) \Delta s \Delta u \\
+ & \int_{t_{1}}^{t} b_{0} q(v) \int_{\sigma(v)}^{\infty} \int_{u}^{\infty} R(s) \Delta s \Delta u \Delta v+\int_{t_{1}}^{t} \omega(v) \int_{v}^{\infty} R(s) \Delta s \Delta v \\
+ & \int_{t_{1}}^{t} \omega^{2}(v) \int_{\sigma(v)}^{\infty} \int_{u}^{\infty} R(s) \Delta s \Delta u \int_{v}^{\infty} R(s) \Delta s \Delta v \leq 0 .
\end{aligned}
$$

We set

$$
A:=\int_{\sigma(v)}^{\infty} \int_{u}^{\infty} R(s) \Delta s \Delta u \int_{v}^{\infty} R(s) \Delta s
$$

and

$$
B:=\int_{v}^{\infty} R(s) \Delta s, \quad y:=-\omega(v) .
$$

Using the inequality

$$
A y^{2}-B y \geq-\frac{B^{2}}{4 A}, \quad A>0,
$$

we obtain

$$
\begin{aligned}
& \omega^{2}(v) \int_{\sigma(v)}^{\infty} \int_{u}^{\infty} R(s) \Delta s \Delta u \int_{v}^{\infty} R(s) \Delta s+\omega(v) \int_{v}^{\infty} R(s) \Delta s \\
\geq & -\frac{\int_{v}^{\infty} R(s) \Delta s}{4 \int_{\sigma(v)}^{\infty} \int_{u}^{\infty} R(s) \Delta s \Delta u} .
\end{aligned}
$$

Using (2.14), we have

$$
\begin{aligned}
& \quad \int_{t_{1}}^{t}\left[b_{0} q(v) \int_{\sigma(v)}^{\infty} \int_{u}^{\infty} R(s) \Delta s \Delta u-\frac{\int_{v}^{\infty} R(s) \Delta s}{4 \int_{\sigma(v)}^{\infty} \int_{u}^{\infty} R(s) \Delta s \Delta u}\right] \Delta v \\
& \leq 1+\omega\left(t_{1}\right) \int_{t_{1}}^{\infty} \int_{u}^{\infty} R(s) \Delta s \Delta u,
\end{aligned}
$$

which contradicts condition (2.1). If $a_{0}=0$, then $\lim _{t \rightarrow \infty} x(t)=0$ clearly.

Assume (2). We define

$$
\varphi(t):=\frac{r(t) z^{\Delta^{3}}(t)}{z^{\Delta^{2}}(t)}, \quad t \in\left[t_{1}, \infty\right)_{\mathbb{T}} .
$$

Then $\varphi(t)<0$ for $t \in\left[t_{1}, \infty\right)_{\mathbb{T}}$ and 


$$
\begin{aligned}
\varphi^{\Delta}(t) & =\frac{\left(r z^{\Delta^{3}}\right)^{\Delta}(t)}{z^{\Delta^{2}}(\sigma(t))}-\frac{r(t)\left(z^{\Delta^{3}}\right)^{2}(t)}{z^{\Delta^{2}}(t) z^{\Delta^{2}}(\sigma(t))} \\
& =-q(t) \frac{x(\delta(t))}{z^{\Delta^{2}}(\sigma(t))}-\frac{r(t)\left(z^{\Delta^{3}}\right)^{2}(t)}{z^{\Delta^{2}}(t) z^{\Delta^{2}}(\sigma(t))} .
\end{aligned}
$$

Recalling that $z>0, z^{\Delta}>0, z^{\Delta^{2}}>0$, and $z^{\Delta^{3}}<0$, from [14, Lemma 4], we have

$$
z(t) \geq d \frac{h_{2}\left(t, t_{0}\right)}{t} z^{\Delta}(t)
$$

for $t \in\left[t_{d}, \infty\right)_{\mathbb{T}}$ and for every $d \in(1 / 2,1)$. On the other hand, we obtain

$$
z^{\Delta}(t)=z^{\Delta}\left(t_{1}\right)+\int_{t_{1}}^{t} z^{\Delta^{2}}(s) \Delta s \geq\left(t-t_{1}\right) z^{\Delta^{2}}(t) \geq \frac{t}{2 d} z^{\Delta^{2}}(t)
$$

for $t \in\left[t_{2}, \infty\right)_{\mathbb{T}}$ sufficiently large. It follows from (2.19) and (2.20) that

$$
z(t) \geq \frac{h_{2}\left(t, t_{0}\right)}{2} z^{\Delta^{2}}(t) .
$$

From $z^{\Delta}>0$ and $\tau(t) \leq t$, we have

$$
x(t) \geq(1-p(t)) z(t) .
$$

In view of (2.21) and (2.22), we get

$$
\begin{aligned}
\frac{x(\delta(t))}{z^{\Delta^{2}}(\sigma(t))} & \geq(1-p(\delta(t))) \frac{z(\delta(t))}{z^{\Delta^{2}}(\delta(t))} \frac{z^{\Delta^{2}}(\delta(t))}{z^{\Delta^{2}}(\sigma(t))} \\
& \geq(1-p(\delta(t))) \frac{h_{2}\left(\delta(t), t_{0}\right)}{2} .
\end{aligned}
$$

Hence, by (2.17) and (2.18), we obtain

$$
\begin{aligned}
\varphi^{\Delta}(t) & \leq-q(t)(1-p(\delta(t))) \frac{h_{2}\left(\delta(t), t_{0}\right)}{2}-\frac{r(t)\left(z^{\Delta^{3}}\right)^{2}(t)}{\left(z^{\Delta^{2}}\right)^{2}(t)} \\
& =-q(t)(1-p(\delta(t))) \frac{h_{2}\left(\delta(t), t_{0}\right)}{2}-\frac{\varphi^{2}(t)}{r(t)} .
\end{aligned}
$$

Since $r z^{\Delta^{3}}$ is decreasing, we have (2.6) using the proof of case (1). Then

$$
\varphi(t) R(t) \geq-1 \text {. }
$$

Multiplying (2.23) by $R(\sigma(t))$ yields

$$
R(\sigma(t)) \varphi^{\Delta}(t) \leq-q(t) R(\sigma(t))(1-p(\delta(t))) \frac{h_{2}\left(\delta(t), t_{0}\right)}{2}-R(\sigma(t)) \frac{\varphi^{2}(t)}{r(t)} .
$$


Integrating the latter inequality from $t_{2}$ to $t$, we have

$$
\begin{aligned}
& R(t) \varphi(t)-R\left(t_{2}\right) \varphi\left(t_{2}\right)+\int_{t_{2}}^{t} q(s) R(\sigma(s))(1-p(\delta(s))) \frac{h_{2}\left(\delta(s), t_{0}\right)}{2} \Delta s \\
+ & \int_{t_{2}}^{t}\left[\frac{\varphi(s)}{r(s)}+R(\sigma(s)) \frac{\varphi^{2}(s)}{r(s)}\right] \Delta s \leq 0 .
\end{aligned}
$$

Now set

$$
A:=\frac{R(\sigma(s))}{r(s)}, \quad B:=\frac{1}{r(s)}, \quad \text { and } \quad y:=-\varphi(s) .
$$

Then, using inequality (2.16), we have

$$
\frac{\varphi(s)}{r(s)}+R(\sigma(s)) \frac{\varphi^{2}(s)}{r(s)} \geq-\frac{1}{4 r(s) R(\sigma(s))} .
$$

Thus, we obtain

$$
\begin{aligned}
\int_{t_{2}}^{t} & {\left[q(s) R(\sigma(s))(1-p(\delta(s))) \frac{h_{2}\left(\delta(s), t_{0}\right)}{2}\right.} \\
& \left.-\frac{1}{4 r(s) R(\sigma(s))}\right] \Delta s \leq 1+R\left(t_{2}\right) \varphi\left(t_{2}\right)
\end{aligned}
$$

due to (2.24), which contradicts condition (2.2).

Assume (3). Recalling that $z^{\Delta^{2}}>0, z^{\Delta^{3}}>0$, and $\left(r z^{\Delta^{3}}\right)^{\Delta}<0$, we have

$$
z^{\Delta^{2}}(t) \geq z^{\Delta^{3}}(t) r(t) \int_{t_{1}}^{t} \frac{1}{r(s)} \Delta s .
$$

Thus, we obtain (see [40, (2.24)])

$$
\left(\frac{z^{\Delta^{2}}}{\int_{t_{1}}^{t} \frac{1}{r(s)} \Delta s}\right)^{\Delta} \leq 0 .
$$

Hence, there exists a $t_{2} \in\left[t_{1}, \infty\right)_{\mathbb{T}}$ such that

$$
\begin{gathered}
z^{\Delta}(t)=z^{\Delta}\left(t_{2}\right)+\int_{t_{2}}^{t} \frac{z^{\Delta^{2}}(s) \int_{t_{1}}^{s} \frac{1}{r(u)} \Delta u}{\int_{t_{1}}^{s} \frac{1}{r(u)} \Delta u} \Delta s \\
\geq \frac{z^{\Delta^{2}}(t)}{\int_{t_{1}}^{t} \frac{1}{r(s)} \Delta s} \int_{t_{2}}^{t} \int_{t_{1}}^{s} \frac{1}{r(u)} \Delta u \Delta s,
\end{gathered}
$$


which implies that

$$
\left(\frac{z^{\Delta}}{\int_{t_{2}}^{t} \int_{t_{1}}^{s} \frac{1}{r(u)} \Delta u \Delta s}\right)^{\Delta} \leq 0 .
$$

Thus, there exists a $t_{3} \in\left[t_{2}, \infty\right)_{\mathbb{T}}$ such that

$$
\begin{aligned}
z(t) & =z\left(t_{3}\right)+\int_{t_{3}}^{t} \frac{z^{\Delta}(s) \int_{t_{2}}^{s} \int_{t_{1}}^{v} \frac{1}{r(u)} \Delta u \Delta v}{\int_{t_{2}}^{s} \int_{t_{1}}^{v} \frac{1}{r(u)} \Delta u \Delta v} \Delta s \\
& \geq \frac{z^{\Delta}(t)}{\int_{t_{2}}^{t} \int_{t_{1}}^{s} \frac{1}{r(u)} \Delta u \Delta s} \int_{t_{3}}^{t} \int_{t_{2}}^{s} \int_{t_{1}}^{v} \frac{1}{r(u)} \Delta u \Delta v \Delta s .
\end{aligned}
$$

It follows from (2.26) and (2.27) that

$$
z(t) \geq \frac{\int_{t_{3}}^{t} \int_{t_{2}}^{s} \int_{t_{1}}^{v} \frac{1}{r(u)} \Delta u \Delta v \Delta s}{\int_{t_{1}}^{t} \frac{1}{r(s)} \Delta s} z^{\Delta^{2}}(t) .
$$

On the other hand, we have (2.22). Then, (2.25) and (2.28) yield

$$
\begin{aligned}
\frac{x(\delta(t))}{z^{\Delta^{2}}(\sigma(t))} & \geq(1-p(\delta(t))) \frac{z(\delta(t))}{z^{\Delta^{2}(\sigma(t))}} \\
& =(1-p(\delta(t))) \frac{z(\delta(t))}{z^{\Delta^{2}(\delta(t))}} \frac{z^{\Delta^{2}}(\delta(t))}{z^{\Delta^{2}}(\sigma(t))} \\
& \geq(1-p(\delta(t))) \frac{\int_{t_{3}}^{\delta(t)} \int_{t_{2}}^{s} \int_{t_{1}}^{v} \frac{1}{r(u)} \Delta u \Delta v \Delta s}{\int_{t_{1}}^{\sigma(t)} \frac{1}{r(s)} \Delta s} .
\end{aligned}
$$

We now set

$$
\psi(t):=\alpha(t) \frac{r(t) z^{\Delta^{3}}(t)}{z^{\Delta^{2}}(t)}, \quad t \in\left[t_{1}, \infty\right)_{\mathbb{T}} .
$$

Then $\psi(t)>0$ for $t \in\left[t_{1}, \infty\right)_{\mathbb{T}}$ and

$$
\begin{aligned}
\psi^{\Delta}(t) & =\alpha^{\Delta}(t) \frac{r(t) z^{\Delta^{3}}(t)}{z^{\Delta^{2}}(t)}+\alpha(\sigma(t))\left(\frac{r z^{\Delta^{3}}}{z^{\Delta^{2}}}\right)^{\Delta}(t) \\
& =\frac{\alpha^{\Delta}(t)}{\alpha(t)} \psi(t)+\alpha(\sigma(t)) \frac{\left(r z^{\Delta^{3}}\right){ }^{\Delta}(t) z^{\Delta^{2}}(t)}{z^{\Delta^{2}}(t) z^{\Delta^{2}}(\sigma(t))}-\alpha(\sigma(t)) \frac{r(t)\left(z^{\Delta^{3}}\right)^{2}(t)}{z^{\Delta^{2}}(t) z^{\Delta^{2}}(\sigma(t))}
\end{aligned}
$$




$$
\leq \frac{\alpha_{+}^{\Delta}(t)}{\alpha(t)} \psi(t)-\alpha(\sigma(t)) q(t) \frac{x(\delta(t))}{z^{\Delta^{2}}(\sigma(t))}-\alpha(\sigma(t)) \frac{r(t)\left(z^{\Delta^{3}}\right)^{2}(t)}{z^{\Delta^{2}}(t) z^{\Delta^{2}}(\sigma(t))} .
$$

Then, we have

$$
\begin{aligned}
\psi^{\Delta}(t) \leq & \frac{\alpha_{+}^{\Delta}(t)}{\alpha(t)} \psi(t)-\alpha(\sigma(t)) q(t) \frac{x(\delta(t))}{z^{\Delta^{2}}(\sigma(t))} \\
& -\alpha(\sigma(t)) \frac{r(t)\left(z^{\Delta^{3}}\right)^{2}(t)}{\left(z^{\Delta^{2}}\right)^{2}(t)} \frac{z^{\Delta^{2}}(t)}{z^{\Delta^{2}}(\sigma(t))} .
\end{aligned}
$$

From (2.25) and (2.30), we obtain

$$
\begin{gathered}
\psi^{\Delta}(t) \leq \frac{\alpha_{+}^{\Delta}(t)}{\alpha(t)} \psi(t)-\alpha(\sigma(t)) q(t) \frac{x(\delta(t))}{z^{\Delta^{2}}(\sigma(t))} \\
-\alpha(\sigma(t)) \frac{\psi^{2}(t)}{\alpha^{2}(t) r(t)} \frac{\int_{t_{1}}^{t} \frac{1}{r(s)} \Delta s}{\int_{t_{1}}^{\sigma(t)} \frac{1}{r(s)} \Delta s} .
\end{gathered}
$$

Now we set

$$
B:=\frac{\alpha_{+}^{\Delta}(t)}{\alpha(t)}, \quad A:=\frac{\alpha(\sigma(t))}{\alpha^{2}(t) r(t)} \frac{\int_{t_{1}}^{t} \frac{1}{r(s)} \Delta s}{\int_{t_{1}}^{\sigma(t)} \frac{1}{r(s)} \Delta s}, \quad \text { and } \quad y:=\psi(t) .
$$

Using the inequality

$$
B y-A y^{2} \leq \frac{B^{2}}{4 A}, \quad A>0
$$

we get

$$
\frac{\alpha_{+}^{\Delta}(t)}{\alpha(t)} \psi(t)-\alpha(\sigma(t)) \frac{\int_{t_{1}}^{t} \frac{1}{r(s)} \Delta s}{\int_{t_{1}}^{\sigma(t)} \frac{1}{r(s)} \Delta s} \frac{\psi^{2}(t)}{\alpha^{2}(t) r(t)} \leq \frac{r(t)\left(\alpha_{+}^{\Delta}(t)\right)^{2}}{4 \alpha(\sigma(t))} \frac{\int_{t_{1}}^{\sigma(t)} \frac{1}{r(s)} \Delta s}{\int_{t_{1}}^{t} \frac{1}{r(s)} \Delta s} .
$$

Thus, we obtain by (2.29) and (2.31) that

$$
\begin{aligned}
\psi^{\Delta}(t) \leq & -\alpha(\sigma(t)) q(t)(1-p(\delta(t))) \frac{\int_{t_{3}}^{\delta(t)} \int_{t_{2}}^{s} \int_{t_{1}}^{v} \frac{1}{r(u)} \Delta u \Delta v \Delta s}{\int_{t_{1}}^{\sigma(t)} \frac{1}{r(s)} \Delta s} \\
+ & \frac{r(t)\left(\alpha_{+}^{\Delta}(t)\right)^{2}}{4 \alpha(\sigma(t))} \frac{\int_{t_{1}}^{\sigma(t)} \frac{1}{r(s)} \Delta s}{\int_{t_{1}}^{t} \frac{1}{r(s)} \Delta s} .
\end{aligned}
$$


Integrating the latter inequality from $t_{4}\left(t_{4} \in\left[t_{3}, \infty\right)_{\mathbb{T}}\right)$ to $t$ yields

$$
\begin{aligned}
\int_{t_{4}}^{t} & {\left[\alpha(\sigma(s)) q(s)(1-p(\delta(s))) \frac{\int_{t_{3}}^{\delta(s)} \int_{t_{2}}^{\eta} \int_{t_{1}}^{v} \frac{1}{r(u)} \Delta u \Delta v \Delta \eta}{\int_{t_{1}}^{\sigma(s)} \frac{1}{r(u)} \Delta u}\right.} \\
& \left.-\frac{r(s)\left(\alpha_{+}^{\Delta}(s)\right)^{2}}{4 \alpha(\sigma(s))} \frac{\int_{t_{1}}^{\sigma(s)} \frac{1}{r(u)} \Delta u}{\int_{t_{1}}^{s} \frac{1}{r(u)} \Delta u}\right] \Delta s \leq \psi\left(t_{4}\right),
\end{aligned}
$$

which contradicts condition (2.3).

Assume (4). From $z>0, z^{\Delta}>0$, and $z^{\Delta^{2}}<0$, we see that

$$
z(t) \geq\left(t-t_{1}\right) z^{\Delta}(t)
$$

and so

$$
\left(\frac{z}{t-t_{1}}\right)^{\Delta} \leq 0
$$

Hence, we have

$$
\frac{z(t)}{z(\sigma(t))} \geq k \frac{t}{\sigma(t)}
$$

and (see [27, Lemma 1])

$$
\frac{z(\delta(t))}{z(t)} \geq k \frac{\delta(t)}{t}
$$

for every $k \in(0,1)$ and for $t \in\left[t_{k}, \infty\right)_{\mathbb{T}}$ sufficiently large. Note that (2.22) holds. Hence, by (1.1) and (2.33), we have

$$
r(v) z^{\Delta^{3}}(v)-r(t) z^{\Delta^{3}}(t)+k z(t) \int_{t}^{v} q(s)(1-p(\delta(s))) \frac{\delta(s)}{s} \Delta s \leq 0 .
$$

Letting $v \rightarrow \infty$ in this inequality, we get

$$
-z^{\Delta^{3}}(t)+k \frac{z(t)}{r(t)} \int_{t}^{\infty} q(s)(1-p(\delta(s))) \frac{\delta(s)}{s} \Delta s \leq 0 .
$$

Thus,

$$
-z^{\Delta^{2}}(v)+z^{\Delta^{2}}(t)+k z(t) \int_{t}^{v} \frac{1}{r(u)} \int_{u}^{\infty} q(s)(1-p(\delta(s))) \frac{\delta(s)}{s} \Delta s \Delta u \leq 0 .
$$

Letting $v \rightarrow \infty$ in this inequality, we have 


$$
z^{\Delta^{2}}(t)+k z(t) \int_{t}^{\infty} \frac{1}{r(u)} \int_{u}^{\infty} q(s)(1-p(\delta(s))) \frac{\delta(s)}{s} \Delta s \Delta u \leq 0 .
$$

Now define

$$
\theta(t):=\beta(t) \frac{z^{\Delta}(t)}{z(t)}, \quad t \in\left[t_{1}, \infty\right)_{\mathbb{T}} .
$$

Then $\theta(t)>0$ for $t \in\left[t_{1}, \infty\right)_{\mathbb{T}}$ and we have

$$
\begin{aligned}
\theta^{\Delta}(t) & =\beta^{\Delta}(t) \frac{z^{\Delta}(t)}{z(t)}+\beta(\sigma(t)) \frac{z^{\Delta^{2}}(t) z(t)-\left(z^{\Delta}\right)^{2}(t)}{z(t) z(\sigma(t))} \\
& =\frac{\beta^{\Delta}(t)}{\beta(t)} \theta(t)+\beta(\sigma(t)) \frac{z^{\Delta^{2}}(t)}{z(\sigma(t))}-\frac{\beta(\sigma(t))}{\beta^{2}(t)} \frac{z(t)}{z(\sigma(t))} \theta^{2}(t)
\end{aligned}
$$

due to (2.35). Thus, by (2.32) and (2.34), we obtain

$$
\begin{aligned}
\theta^{\Delta}(t) \leq & -k^{2} \beta(\sigma(t)) \frac{t}{\sigma(t)} \int_{t}^{\infty} \frac{1}{r(u)} \int_{u}^{\infty} q(s)(1-p(\delta(s))) \frac{\delta(s)}{s} \Delta s \Delta u \\
& +\frac{\beta_{+}^{\Delta}(t)}{\beta(t)} \theta(t)-k \frac{\beta(\sigma(t))}{\beta^{2}(t)} \frac{t}{\sigma(t)} \theta^{2}(t) .
\end{aligned}
$$

Hence,

$$
\begin{aligned}
\theta^{\Delta}(t) \leq & -k^{2} \beta(\sigma(t)) \frac{t}{\sigma(t)} \int_{t}^{\infty} \frac{1}{r(u)} \int_{u}^{\infty} q(s)(1-p(\delta(s))) \frac{\delta(s)}{s} \Delta s \Delta u \\
& +\frac{\sigma(t)\left(\beta_{+}^{\Delta}(t)\right)^{2}}{4 k t \beta(\sigma(t))} .
\end{aligned}
$$

Integrating the latter inequality from $t_{k}$ to $t$ implies that

$$
\begin{aligned}
\int_{t_{k}}^{t} & {\left[k^{2} \beta(\sigma(s)) \frac{s}{\sigma(s)} \int_{s}^{\infty} \frac{1}{r(u)} \int_{u}^{\infty} q(v)(1-p(\delta(v))) \frac{\delta(v)}{v} \Delta v \Delta u\right.} \\
& \left.-\frac{\sigma(s)\left(\beta_{+}^{\Delta}(s)\right)^{2}}{4 k s \beta(\sigma(s))}\right] \Delta s \leq \theta\left(t_{k}\right)
\end{aligned}
$$

holds for every $k \in(0,1)$, which contradicts condition (2.4). The proof is complete.

\section{EXAMPLE AND Discussion}

The following example illustrates applications of theoretical results in the previous section. 
Example 3.1. Consider a fourth-order neutral delay dynamic equation

$$
\left(t^{4}\left(x(t)+\frac{1}{2} x\left(\frac{t}{2}\right)\right)^{\Delta^{3}}\right)^{\Delta}+\lambda t x\left(\frac{t}{2}\right)=0,
$$

where $t \in \mathbb{T}:=\overline{2^{\mathbb{Z}}}:=\left\{2^{k}: k \in \mathbb{Z}\right\} \cup\{0\}$ and $\lambda>0$ is a constant. Let $\gamma=1$ and $r(t)=t^{4}$. Then

$$
R(t)=\frac{8}{7 t^{3}}, \quad \int_{u}^{\infty} R(s) \Delta s=\frac{32}{21 u^{2}}, \quad \text { and } \quad \int_{v}^{\infty} \int_{u}^{\infty} R(s) \Delta s \Delta u=\frac{64}{21 v} .
$$

Using [9, Example 1.104], we have

$$
h_{2}\left(t, t_{0}\right)=\frac{\left(t-t_{0}\right)\left(t-2 t_{0}\right)}{3}
$$

and so

$$
h_{2}\left(\delta(t), t_{0}\right)=h_{2}\left(\frac{t}{2}, t_{0}\right)=\frac{\left(\frac{t}{2}-t_{0}\right)\left(\frac{t}{2}-2 t_{0}\right)}{3} \geq \frac{t^{2}}{13}
$$

for all sufficiently large $t$. It is easy to verify that all assumptions of Theorem 2.2 are satisfied, if $\alpha(t)=\beta(t)=1$. Hence, every solution $x$ of (3.1) is either oscillatory or satisfies $\lim _{t \rightarrow \infty} x(t)=0$.

Remark 3.2. Most oscillation results reported in the literature (see, e.g., $[1,13,20$ 22]) for the neutral dynamic equation (1.1) and its particular cases have been obtained under the case where $\int_{t_{0}}^{\infty} \frac{\Delta t}{r(t)}=\infty$ which significantly simplifies the analysis of the behavior of $z:=x+p(x \circ \tau)$ for a nonoscillatory solution $x$ of (1.1). In this paper, using Riccati transformation technique, we obtain a new asymptotic criterion for the fourth-order neutral delay dynamic equation (1.1) with noncanonical operators (i.e., (1.2) is satisfied).

Remark 3.3. We stress that the study of asymptotic behavior of equation (1.1) in the case (1.2) brings additional difficulties. In particular, in order to deal with the case where $z^{\Delta}<0$ (which is simply eliminated if $\int_{t_{0}}^{\infty} \frac{\Delta t}{r(t)}=\infty$ holds when using a proof similar to that of [1, Lemma 2.2]), the arbitrariness in the choice of $b_{0}$ is required. As a matter of fact, it is well known (see, e.g., $[13,22]$ ) that if $x$ is an eventually positive solution of (1.1), then (2.22) is satisfied. One of the principal difficulties one encounters lies in the fact that (2.22) does not hold when (1.2) is satisfied.

Remark 3.4. Since the signs of $z^{\Delta}, z^{\Delta^{2}}$, and $z^{\Delta^{3}}$ have four possible cases, our criterion for asymptotic behavior of (1.1), as in [40, Theorem 2.1], includes four assumptions. It would be of interest to find another method to study (1.1) in order to simplify these conditions. 


\section{ACKNOWLEDGMENTS}

The authors express their sincere gratitude to the editors and anonymous referee for the careful reading of the original manuscript and useful comments that helped to improve the presentation of the results and accentuate important details. This research is supported by National Key Basic Research Program of P. R. China (2013CB035604) and NNSF of P. R. China (Grant Nos. 61034007, 51277116, 51107069).

\section{REFERENCES}

1. R. P. Agarwal, M. Bohner, T. Li and C. Zhang, Oscillation theorems for fourth-order half-linear delay dynamic equations with damping, Mediterr. J. Math., (2013), in press.

2. R. P. Agarwal, M. Bohner and S. H. Saker, Oscillation of second order delay dynamic equations, Can. Appl. Math. Q., 13 (2005), 1-17.

3. R. P. Agarwal, M. Bohner, S. Tang, T. Li and C. Zhang, Oscillation and asymptotic behavior of third-order nonlinear retarded dynamic equations, Appl. Math. Comput., 219 (2012), 3600-3609.

4. R. P. Agarwal, D. O’Regan and S. H. Saker, Oscillation criteria for second-order nonlinear neutral delay dynamic equations, J. Math. Anal. Appl., 300 (2004), 203-217.

5. E. Akın-Bohner, M. Bohner and S. H. Saker, Oscillation criteria for a certain class of second order Emden-Fowler dynamic equations, Electron. Trans. Numer. Anal., 27 (2007), 1-12.

6. D. R. Anderson, A fourth-order nonlinear difference equation, J. Difference Equ. Appl., 9 (2003), 161-169.

7. B. Baculíková and J. Džurina, Oscillation of third-order neutral differential equations, Math. Comput. Modelling, 52 (2010), 215-226.

8. M. Bartušek, M. Cecchi, Z. Došlá and M. Marini, Fourth-order differential equation with deviating argument, Abstr. Appl. Anal., 2012 (2012), 1-17.

9. M. Bohner and A. Peterson, Dynamic Equations on Time Scales: An Introduction with Applications, Birkhäuser, Boston, 2001.

10. M. Bohner and A. Peterson, Advances in Dynamic Equations on Time Scales, Birkhäuser, Boston, 2003.

11. M. Bohner and S. H. Saker, Oscillation of second order nonlinear dynamic equations on time scales, Rocky Mt. J. Math., 34 (2004), 1239-1254.

12. Z. Došlá and J. Krejčová, Oscillation of a class of the fourth-order nonlinear difference equations, Adv. Difference Equ., 2012 (2012), 1-14.

13. L. Erbe, B. Karpuz and A. Peterson, Kamenev-type oscillation criteria for higher-order neutral delay dynamic equations, Int. J. Difference Equ., 6 (2011), 1-16. 
14. L. Erbe, A. Peterson and S. H. Saker, Hille and Nehari type criteria for third-order dynamic equations, J. Math. Anal. Appl., 329 (2007), 112-131.

15. S. R. Grace, R. P. Agarwal and S. Pinelas, On the oscillation of fourth order superlinear dynamic equations on time scales, Dyn. Syst. Appl., 20 (2011), 45-54.

16. S. R. Grace, R. P. Agarwal and W. Sae-jie, Monotone and oscillatory behavior of certain fourth order nonlinear dynamic equations, Dyn. Syst. Appl., 19 (2010), 25-32.

17. S. R. Grace, M. Bohner and S. Sun, Oscillation of fourth-order dynamic equations, Hacet. J. Math. Stat., 39 (2010), 545-553.

18. J. R. Graef, S. Panigrahi and P. Rami Reddy, Oscillation results for fourth-order nonlinear neutral dynamic equations, Commun. Math. Anal., 15 (2013), 11-28.

19. S. Hilger, Analysis on measure chains-a unified approach to continuous and discrete calculus, Results Math., 18 (1990), 18-56.

20. B. Karpuz, Unbounded oscillation of higher-order nonlinear delay dynamic equations of neutral type with oscillating coefficients, Electron. J. Qual. Theory Differ. Equ., 34 (2009), 1-14.

21. B. Karpuz, Asymptotic behaviour of bounded solutions of a class of higher-order neutral dynamic equations, Appl. Math. Comput., 215 (2009), 2174-2183.

22. B. Karpuz, Sufficient conditions for the oscillation and asymptotic behaviour of higherorder dynamic equations of neutral type, Appl. Math. Comput., 221 (2013), 453-462.

23. T. Li, R. P. Agarwal and M. Bohner, Some oscillation results for second-order neutral dynamic equations, Hacet. J. Math. Stat., 41 (2012), 715-721.

24. T. Li, Z. Han, S. Sun and Y. Zhao, Oscillation results for third order nonlinear delay dynamic equations on time scales, Bull. Malays. Math. Sci. Soc., 34 (2011), 639-648.

25. T. Li, E. Thandapani and S. Tang, Oscillation theorems for fourth-order delay dynamic equations on time scales, Bull. Math. Anal. Appl., 3 (2011), 190-199.

26. S. Panigrahi and P. Rami Reddy, On oscillatory and asymptotic behavior of fourth order non-linear neutral delay dynamic equations, Comput. Math. Appl., 62 (2011), 4258-4271.

27. Y. Şahiner, Oscillation of second-order delay differential equations on time scales, Nonlinear Anal. TMA, 63 (2005), 1073-1080.

28. S. H. Saker, Oscillation of nonlinear dynamic equations on time scales, Appl. Math. Comput., 148 (2004), 81-91.

29. S. H. Saker, Oscillation Theory of Dynamic Equations on Time Scales, Lambert Academic Publisher, Germany, 2010.

30. S. H. Saker, On oscillation of a certain class of third-order nonlinear functional dynamic equations on time scales, Bull. Math. Soc. Sci. Math. Roum., 54 (2011), 365-389.

31. S. H. Saker, Oscillation of third-order functional dynamic equations on time scales, Sci. China Math., 54 (2011), 2597-2614. 
32. S. H. Saker and J. R. Graef, Oscillation of third-order nonlinear neutral functional dynamic equations on time scales, Dyn. Syst. Appl., 21 (2012), 583-606.

33. S. H. Saker and D. O’Regan, New oscillation criteria for second-order neutral functional dynamic equations via the generalized Riccati substitution, Commun. Nonlinear Sci. Numer. Simul., 16 (2011), 423-434.

34. E. Thandapani and I. M. Arockiasamy, On fourth order nonlinear oscillations of difference equations, Comput. Math. Appl., 42 (2001), 357-368.

35. E. Thandapani, J. R. Graef and P. W. Spikes, On existence of positive solutions and oscillations of neutral difference equations of odd order, J. Difference Equ. Appl., 2 (1996), 175-183.

36. E. Thandapani, S. Pandian, R. Dhanasekaran and J. R. Graef, Asymptotic results for a class of fourth order quasilinear difference equations, J. Difference Equ. Appl., 13 (2007), 1085-1103.

37. E. Thandapani, V. Piramanantham and S. Pinelas, Oscillation theorems of fourth order nonlinear dynamic equations on time scales, Int. J. Pure Appl. Math., 76 (2012), 455-468.

38. X. Wu, T. Sun, H. Xi and C. Chen, Oscillation criteria for fourth-order nonlinear dynamic equations on time scales, Abstr. Appl. Anal., 2013 (2013), 1-11.

39. C. Zhang, R. P. Agarwal, M. Bohner and T. Li, New results for oscillatory behavior of even-order half-linear delay differential equations, Appl. Math. Lett., 26 (2013), 179-183.

40. C. Zhang, T. Li, R. P. Agarwal and M. Bohner, Oscillation results for fourth-order nonlinear dynamic equations, Appl. Math. Lett., 25 (2012), 2058-2065.

41. C. Zhang, T. Li, B. Sun and E. Thandapani, On the oscillation of higher-order half-linear delay differential equations, Appl. Math. Lett., 24 (2011), 1618-1621.

Tongxing Li and Chenghui Zhang

Shandong University

School of Control Science and Engineering

Jinan, Shandong 250061

P. R. China

E-mail: litongx2007@163.com zchui@sdu.edu.cn

Ethiraju Thandapani

University of Madras

Ramanujan Institute for Advanced Study in Mathematics

Chennai, 600 005, India

E-mail: ethandapani@yahoo.co.in 